\begin{tabular}{|c|l|}
\hline Title & Cryogenic receiver front-end with sharp skirt characteristics \\
\hline Author(s) & Narahashi, S.; Satoh, K.; Kawai, K.; Koizumi, D.; Nojima, T. \\
\hline Citation & $\begin{array}{l}\text { Superconductor Science and Technology, 19(5), S416-S422 } \\
\text { https://doi.org/10.1088/0953 2048/19/5/5533 }\end{array}$ \\
\hline Issue Date & 2006-05 \\
\hline Doc URL & http://hdl.handle.net/2115/14603 \\
\hline Rights & Copyright @ Institute of Physics and IOP Publishing Limited 2006. \\
\hline Type & article (author version) \\
\hline File Information & sust6_5_S53 (f揭載ファイル) .pdf \\
\hline
\end{tabular}

Instructions for use 


\title{
Cryogenic receiver front-end with sharp skirt characteristics
}

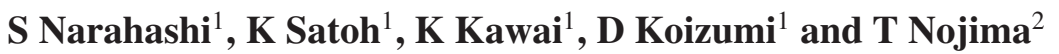 \\ ${ }^{1}$ RF Technology Laboratory, Wireless Laboratories, NTT DoCoMo, Inc, Yokosuka, \\ Kanagawa 239-8536, Japan \\ ${ }^{2}$ Graduate School of Information Science and Technology, Hokkaido University, Sapporo, \\ Hokkaido 060-0808, Japan
}

Received 3 October 2005, in final form 27 February 2006

Published 23 March 2006

Online at stacks.iop.org/SUST/19/S416

\begin{abstract}
This paper presents an experimental cryogenic receiver front-end (CRFE) with sharp skirt characteristics for mobile base stations. The CRFE comprises a high-temperature superconducting filter, a cryogenic low-noise amplifier, and a highly reliable cryostat that is very compact. The major characteristics of the proposed CRFE measured at $70 \mathrm{~K}$ are a centre frequency of $1.95 \mathrm{GHz}$, passband width of $20 \mathrm{MHz}$, sharp selectivity of $20 \mathrm{~dB} / 100 \mathrm{kHz}, 1.4 \mathrm{~dB}$ ripple, $31.3 \mathrm{~dB}$ average passband gain, and average passband equivalent noise temperature of $47.9 \mathrm{~K}$. The CRFE weighs $19 \mathrm{~kg}$ and occupies 351 . Random failure of the cryostat is also evaluated by a continuous operation test using four identical ones simultaneously. The cryostat used in the CRFE has a high reliability level of over five years of continuous maintenance-free operation.
\end{abstract}

(Some figures in this article are in colour only in the electronic version)

\section{Introduction}

With the growing demand for high-speed and high-capacity data transmission in mobile communications, the frequency bands used in mobile radios have become higher and higher. In Japan, the W-CDMA (Wideband CDMA) system, one of the third generation mobile communication systems called 'International Mobile Telecommunications 2000 (IMT-2000)', has been in commercial service since 2001 using the $2 \mathrm{GHz}$ band. It is of great importance to improve the sensitivity of the base station receiver systems because propagation and feeder losses in the $2 \mathrm{GHz}$ band are greater than those in the 800 or $900 \mathrm{MHz}$ band for the second generation mobile communication systems. The technical key to improving the sensitivity is the employment of an extremely low noise figure and high gain receiver front-end composed of receiving bandpass filter and amplifier. It is also important for base station receiver systems to have high selectivity from the standpoint of efficient frequency utilization. This is because the frequency bands used by mobile communication system operators should be assigned as closely as possible without interfering with each other. The most effective approach to suppressing the interference from signals of adjacent bands is to employ a very sharp skirt receiving bandpass filter based on ultra-high- $Q$ material.
High-temperature superconducting filters (HTSFs) have been proposed for use in mobile base station receivers for the purpose of efficient frequency utilization and improving the receiver sensitivity, since HTSFs achieve low insertion loss and sharp skirt characteristics. High frequency selectivity characteristics can also reduce the saturation power level required for the cryogenic low-noise amplifier (CLNA) used in the base stations, since undesired interference signals in the adjacent passband can be thoroughly suppressed, resulting in mitigation of the cryostat cooling capability.

A cryogenic receiver front-end (CRFE), comprising an HTSF, a CLNA, and a highly reliable, compact-sized cryostat, is expected to be an effective and practical way to achieve efficient frequency utilization and high frequency selectivity characteristics for mobile base station receivers [1-3]. The influence of antenna noise, such as ground noise and manmade noise, is evaluated through field tests both in urban and suburban areas [3].

This paper describes the fundamental configuration and characteristics of an experimental CRFE for the $2 \mathrm{GHz}$ band together with characteristics of each component. The experimental CRFE attains sharper characteristics and reduces its volume by $60 \%$ compared with the previously proposed one [3] by increasing the number of the poles of the HTSF and by employing a small-sized cryostat. 


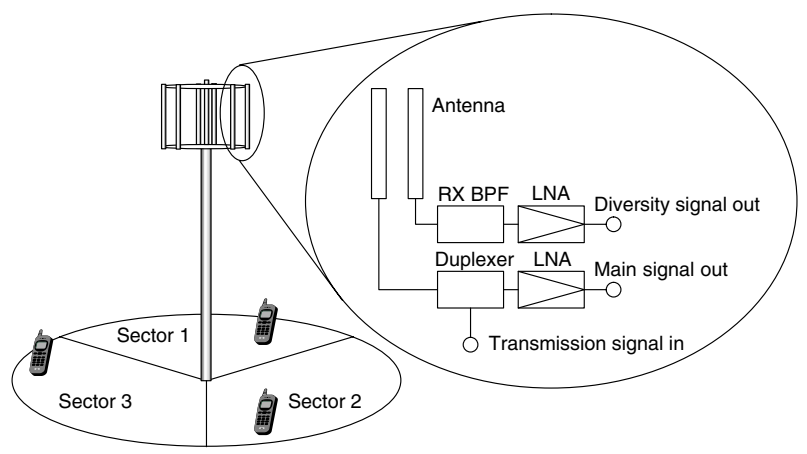

Figure 1. Basic configuration of a three-sector cellular base station receiver system.

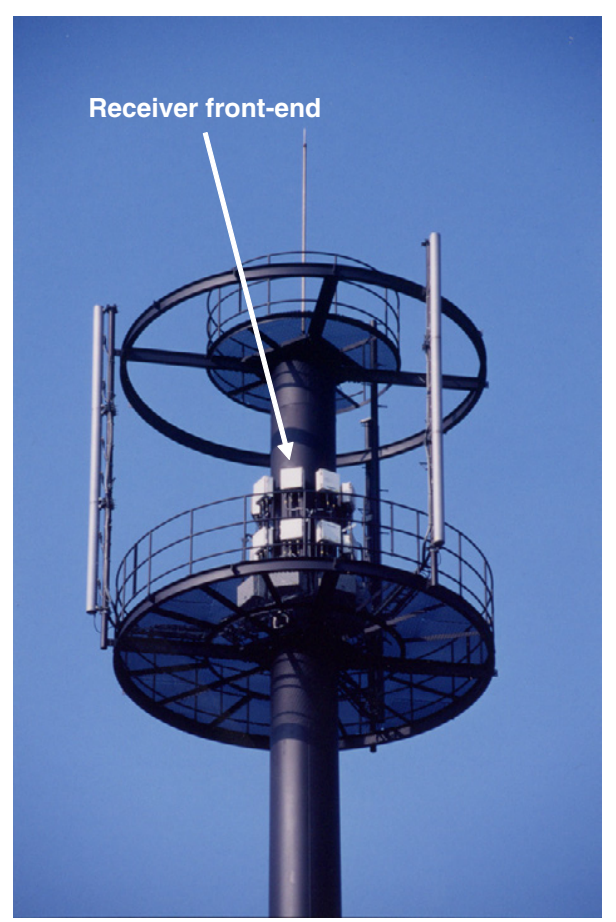

Figure 2. An example of a tower-mounted receiver front-end.

\section{Cryogenic receiver front-end}

\subsection{Fundamental configuration}

Figure 1 shows the basic configuration of a three-sector cellular base station receiver front-end including antennas. Each sector has two antennas (for main and diversity reception). Each antenna is followed by an RF front-end comprising a receiving bandpass filter (RX-BPF) and low-noise amplifier (LNA). The diversity front-end has a simple RX-BPF, while the main frontend uses a duplexer in order to use the same antenna for simultaneous reception and transmission. This receiver frontend is located in the vicinity of the antenna (on top of the tower or tower-mounted), which yields highly sensitive reception. Figure 2 shows an example of an existing tower-mounted receiver front-end. In base station receiver systems, the CRFE mounted on top of the tower appears to be the most promising approach to maximize the sensitivity and efficient frequency utilization.

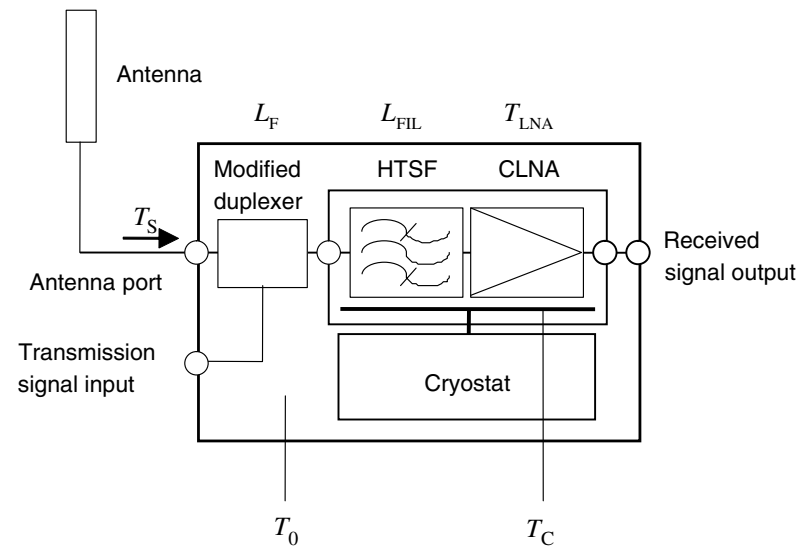

Figure 3. Fundamental configuration of the CRFE.

Figure 3 shows the fundamental configuration of the proposed CRFE. The CRFE comprises an HTSF, CLNA, and cryostat. In this figure, a modified duplexer is inserted in front of the HTSF. The out-of-band attenuation of the receiving band of the modified duplexer is not as large as that of the conventional one shown in figure 1, since the following HTSF provides sufficient performance as an RX-BPF. The CRFE should be lightweight, small, and highly reliable to offer easy installation and maintenance because it is to be installed on the tower-top, similarly to the conventional receiver front-end. The characteristics of each component are described in section 3 .

\subsection{Improvement in sensitivity [3]}

Here, sensitivity stands for the minimum receiving signal power level required to successfully establish a radio communication link between mobile and base stations. Thermal noise evaluation is indispensable in estimating sensitivity because the sensitivity increases as the thermal noise is reduced. The equivalent noise temperature [4] is introduced as an index for evaluating the thermal noise instead of the noise figure. In figure 3 , the total loss from the antenna port to the HTSF input is defined as $L_{\mathrm{F}}$. From [1], the equivalent noise temperature of the CRFE, $T_{\mathrm{R}}$, is given by

$$
T_{\mathrm{R}}=\left(L_{\mathrm{F}}-1\right) T_{0}+L_{\mathrm{F}}\left(L_{\mathrm{FIL}}-1\right) T_{\mathrm{C}}+L_{\mathrm{F}} L_{\mathrm{FIL}} T_{\mathrm{LNA}}
$$

where $L_{\mathrm{FIL}}$ is the HTSF loss; $T_{\mathrm{LNA}}$ is the equivalent noise temperature of the LNA; $T_{0}$ is ambient normal temperature; $T_{\mathrm{C}}$ is the cooled device temperature. $L_{\mathrm{F}}$ and $L_{\mathrm{FIL}}$ are antilogarithms. The contribution of the following equipment (connected to CRFE at a received signal output port) on $T_{\mathrm{R}}$ can be neglected if the CLNA has sufficient amplification gain (over $30 \mathrm{~dB}$ ). Assuming that the equivalent noise temperature of antenna noise at the antenna port in figure 3 (hereinafter referred to as antenna noise temperature) is defined as $T_{\mathrm{S}}$, the total equivalent noise temperature of the CRFE and antenna is given as $T_{\mathrm{R}}+T_{\mathrm{S}}$. When the CRFE is utilized in place of the normal temperature front end, the improvement in sensitivity achieved can be estimated from the total equivalent noise temperature ratio given as

$$
\frac{T_{\mathrm{R} 0}+T_{\mathrm{S}}}{T_{\mathrm{R}}+T_{\mathrm{S}}}
$$




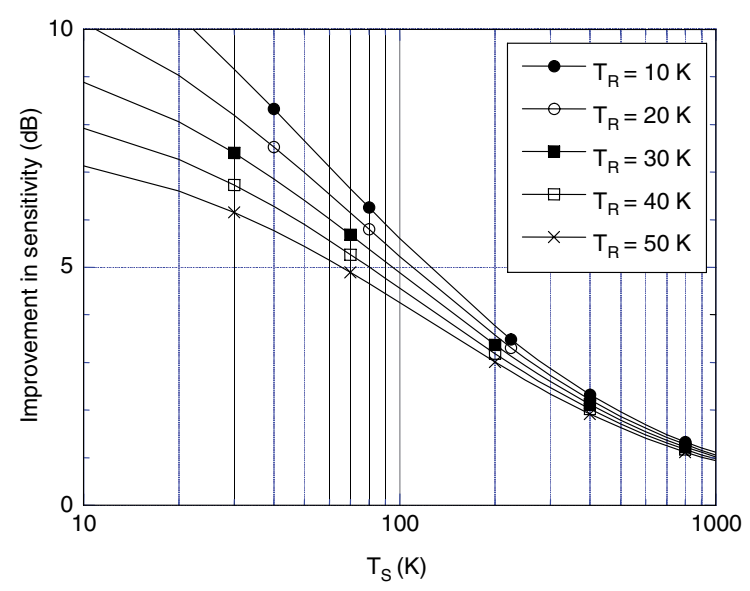

Figure 4. Improvement in the sensitivity of CRFE.

where $T_{\mathrm{R} 0}$ is the equivalent noise temperature of the normal temperature front-end. The estimated $T_{\mathrm{R} 0}$ in the $2 \mathrm{GHz}$ band is typically $300 \mathrm{~K}$ [5]. Figure 4 shows the calculated improvement in sensitivity. In traditional radio system design, $T_{\mathrm{S}}$ is usually set to $300 \mathrm{~K}$ for microwave bands. In this case, the improvement in sensitivity is estimated to exceed $2.5 \mathrm{~dB}$ if $T_{\mathrm{R}}$ is less than $40 \mathrm{~K}$. Actual mobile base station antennas do not necessarily reach $300 \mathrm{~K}$, since the antenna covers some of the sky as well as the ground plane. This is because the equivalent noise temperature for the sky can be assumed to nearly equal to $0 \mathrm{~K}$. $T_{\mathrm{S}}$ can be regarded as $150 \mathrm{~K}$ for the colinear array antennas used widely in current base stations if the antenna ohmic loss is negligibly small. Sensitivity improvements of up to $3 \mathrm{~dB}$ can be expected by employing an antenna with small ohmic loss and a very low-noise CRFE, since actual antenna noise temperatures measured in the field lie between 255 and $272 \mathrm{~K}$ [1].

\subsection{Improvement in selectivity}

As described in section 1, the frequency bands used by the mobile communication system operators should be assigned as closely as possible without interfering with each other. However, undesired interference occurs and it has a negative impact on communication quality unless the RF front-end has high selectivity. Figure 5 shows an example of interference between two mobile communication systems. In the base station receiving system of operator ' $A$ ', the signal from the subscriber unit employing frequency $f_{\mathrm{A}}$ must be passed through the receiving band-pass filter with extremely low loss, and the signal from the subscriber unit of operator 'B', which uses frequency $f_{\mathrm{B}}$, must be suppressed as much as possible. If the base station receiving system of operator ' $A$ ' has low selectivity, in other words, its receiving band pass filter does not offer a very sharp skirt, intermodulation distortion is generated between the frequencies $f_{\mathrm{A}}$ and $f_{\mathrm{B}}$ at the base station receiving system of operator ' $A$ '. However, if the base station receiving system has high selectivity, intermodulation distortion generation is virtually eliminated.

\section{Fundamental characteristics of each component}

\subsection{High-temperature superconducting filter}

The CRFE employs a 22-pole, thin-film HTSF using YBCO (yttrium barium copper oxide). The previously proposed CRFE also employed a thin-film HTSF using YBCO; however, the number of poles is eight. Figure 6 presents the frequency response of the HTSF. The HTSF offers far steeper skirt performance, which is comparable to that previously reported [6] for mobile communications. HTSF usage facilitates the required saturation level of the CLNA since the input of adjacent signals to the CLNA is minimized by the sharp skirt performance. Figure 7 shows the insertion loss of the HTSF at the centre frequency of $1.95 \mathrm{GHz}$. The HTSF

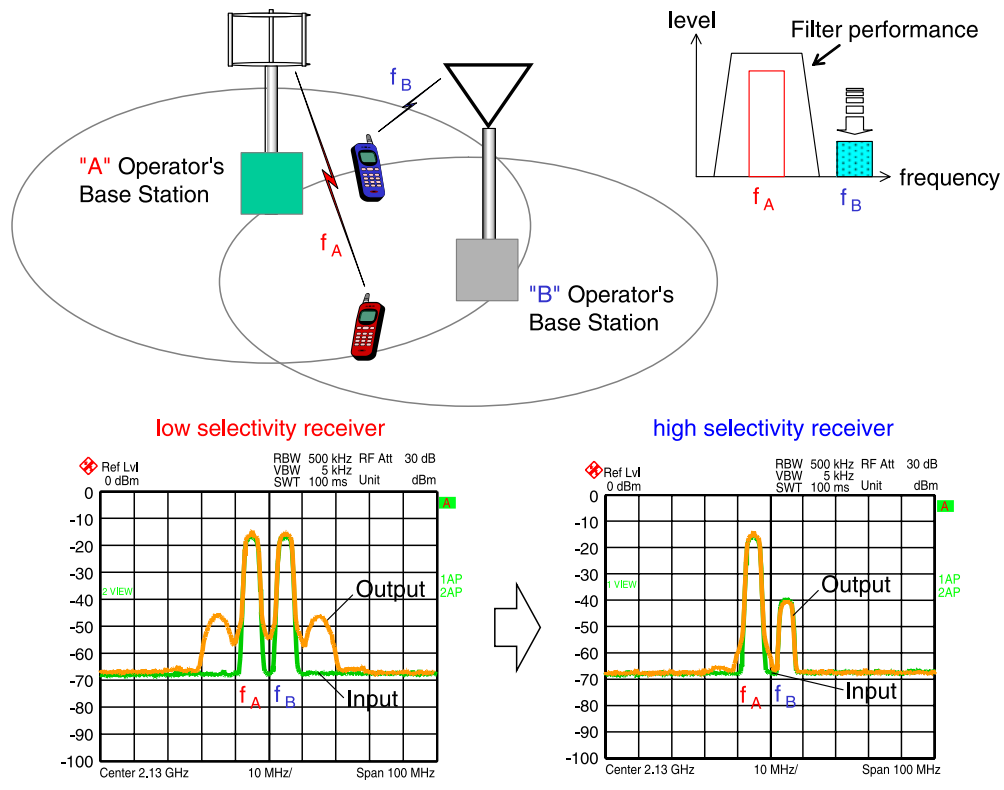

Figure 5. Example of interference between two mobile communication systems. 
Cryogenic receiver front-end with sharp skirt characteristics

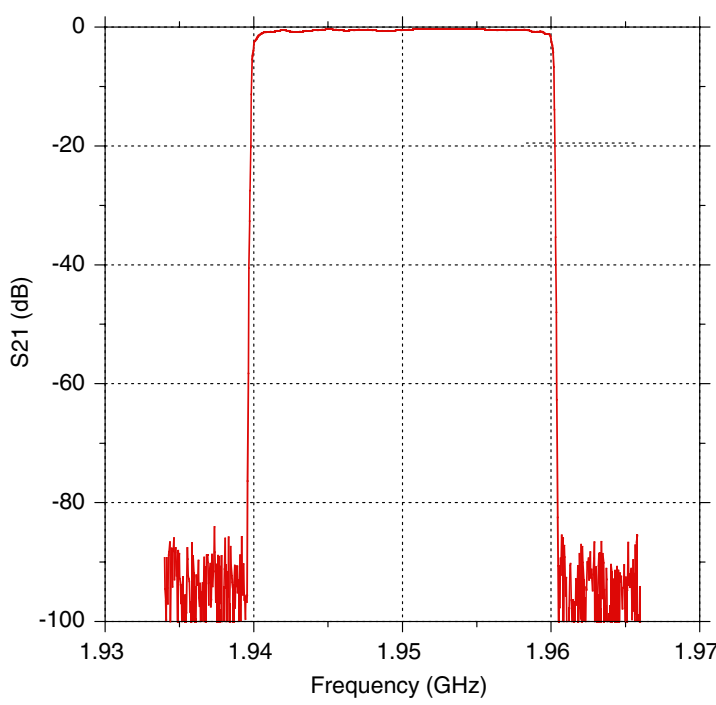

Figure 6. Frequency response of the HTSF.

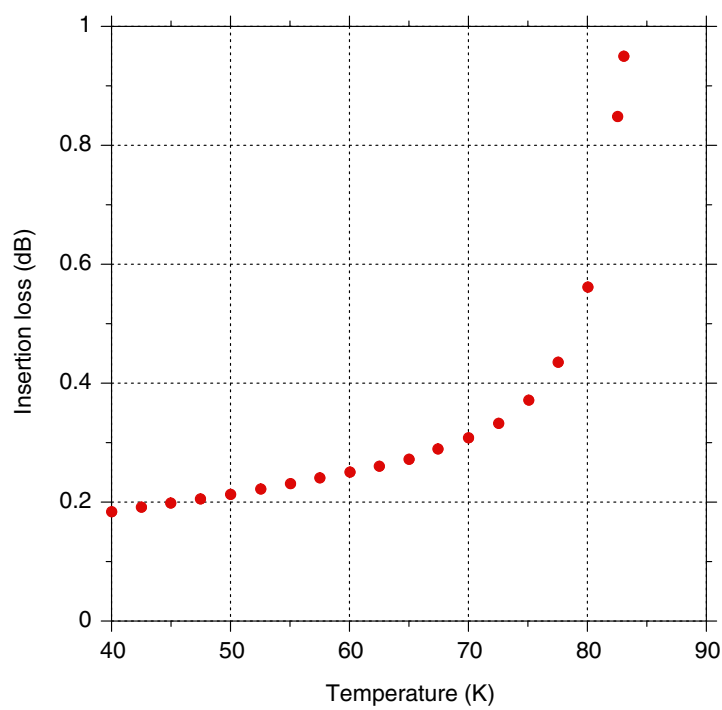

Figure 7. Insertion loss of the HTSF at $1.95 \mathrm{GHz}$.

has a loss of $0.3 \mathrm{~dB}$ at $70 \mathrm{~K}$ including input/output connector losses. A large capacity cryostat is required to attain the lower operating temperature, although the insertion loss decreases as the temperature drops. Figure 8 presents the frequency stability of the HTSF. Band edge frequencies of $3 \mathrm{~dB}$ (lower and upper edges) were measured, and drift frequencies $(\Delta f)$ from the frequencies at $70 \mathrm{~K}\left(f_{0}\right)$ are plotted as normalized frequency deviation $\left(\Delta f / f_{0}\right)$. The edge frequencies shift almost $50 \mathrm{ppm}$ below $70 \mathrm{~K}$ and $80 \mathrm{ppm}$ over $70 \mathrm{~K}$ with a temperature change of $1 \mathrm{~K}$, whereas the bandwidth is quite stable.

\subsection{Cryogenic low-noise amplifier}

A novel $2 \mathrm{GHz}$ band CLNA was developed based on the cryogenic amplifier construction technologies used in radio astronomy [7]. The developed CLNA has a two-stage configuration of an HEMT (high electron mobility transistor)

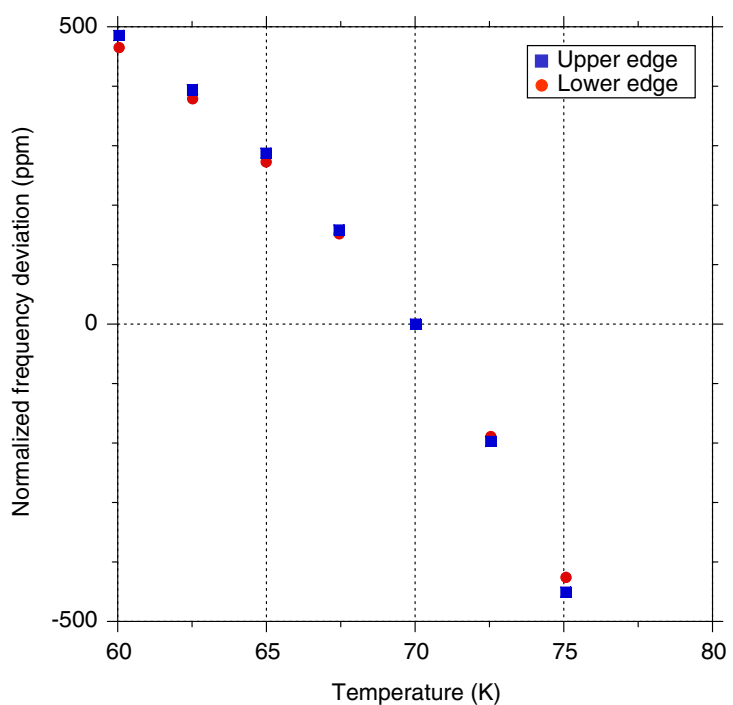

Figure 8. Frequency stability of the HTSF.

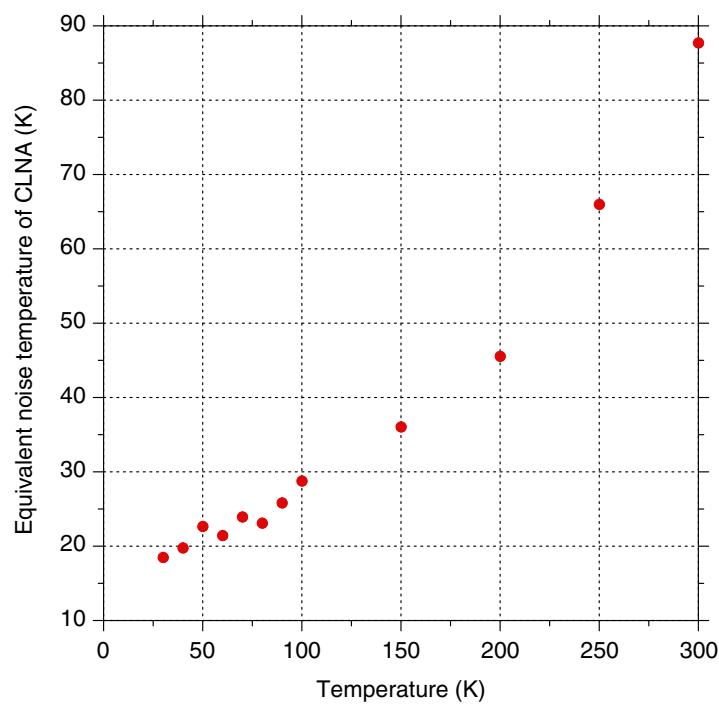

Figure 9. Equivalent noise temperature versus temperature of the CLNA.

and a GaAs-FET; its amplification gain and saturation power are $30 \mathrm{~dB}$ and $25 \mathrm{dBm}$, respectively. An HEMT is used as the first stage because of its low noise characteristic, and a GaAs-FET is used as the second stage because of its high linearity. Even at normal temperature, HEMTs are often exploited to achieve low-noise amplification. The equivalent noise temperature of HEMTs decreases almost quadratically by cooling them [8], whereas the equivalent noise temperature of GaAs-FETs decreases almost linearly as they are cooled [9]. Figure 9 presents the equivalent noise temperature of the developed CLNA. The CLNA achieves the equivalent noise temperature of $25 \mathrm{~K}$ (corresponding to a noise figure of $\mathrm{NF}=$ $0.3 \mathrm{~dB}$ ) or less when the cooling temperature of the CLNA is below $75 \mathrm{~K}$. 


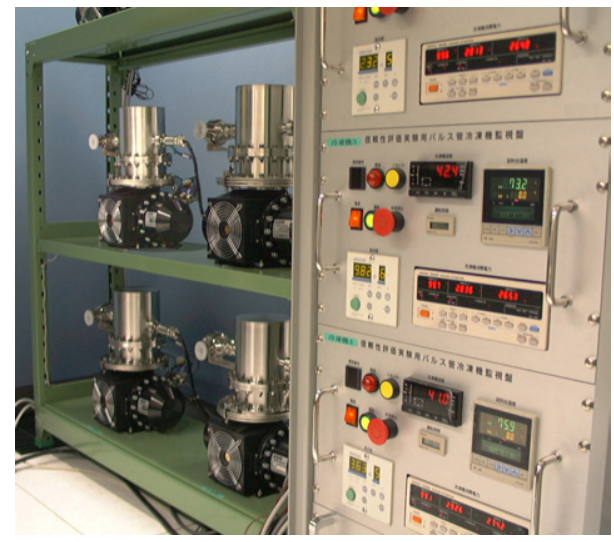

Figure 10. Continuous operation test for evaluating random failure with four identical cryostats.

\subsection{Cryostat}

The cryostat used in the CRFE is a key device for miniaturization. The proposed CRFE employs a newly developed cryostat that takes advantage of the high reliability, high power-efficiency, and compactness of the Stirling-type pulse tube. The cooling capability is set to be $2 \mathrm{~W}$ at $77 \mathrm{~K}$ to achieve a lightweight CRFE. The cryostat mainly comprises a Stirling-type non-sliding compressor [10] and a pulse-tube expander. This type of cryostat has no moving parts, so an infinite service life may be anticipated, while the service life of a conventional cryostat [11] is limited due to seal deterioration. Only random failure need be considered. This random failure has been evaluated by a continuous-operation test using four of the same cryostats simultaneously as shown in figure 10 .

If the cryostat has no failure over time interval $T$, the lower bound of the confidence level for the mean time to failure (MTTF) of the cryostat obeys the following equation:

$$
(\mathrm{MTTF})_{\mathrm{L}}=\frac{2 T}{\chi^{2}(2, \alpha)}
$$

Here, the denominator of equation (3) stands for the chisquare distribution where the number of degrees of freedom is 2 and the confidence level is $\alpha$. In this evaluation test, the target MTTF was set to $40000 \mathrm{~h}$, approximately five years. Figure 11 shows the durability of the cryostat. The total operating time, over $170000 \mathrm{~h}$, has been achieved to date with no failure. The confidence level was $98 \%$ on 31 August 2005. This means that 98 out of 100 cryostats achieve the MTTF of $40000 \mathrm{~h}$. These results suggest that the cryostat has a high reliability level of over five years of continuous maintenance-free operation.

\subsection{Duplexer}

As described in section 2, the mobile base station system requires a duplexer for the purpose of exploiting one antenna for both receiving and transmitting. It is difficult to construct a duplexer using superconducting material. This is because intermodulation distortion components are generated if high power transmitting (TX) band signals are input to the HTSF. The CRFE employs a dielectric modified duplexer in order to decrease the TX band signal power to the HTSF and to

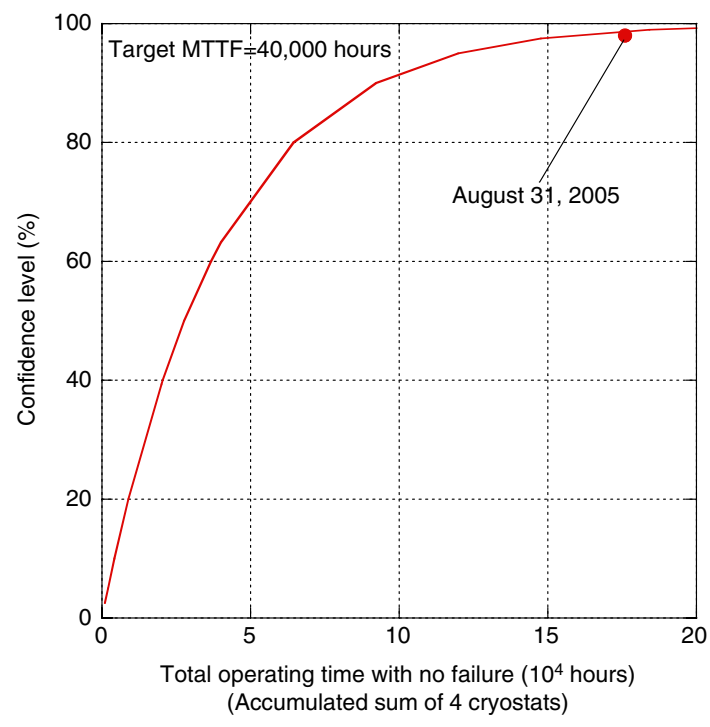

Figure 11. Durability of the cryostat.

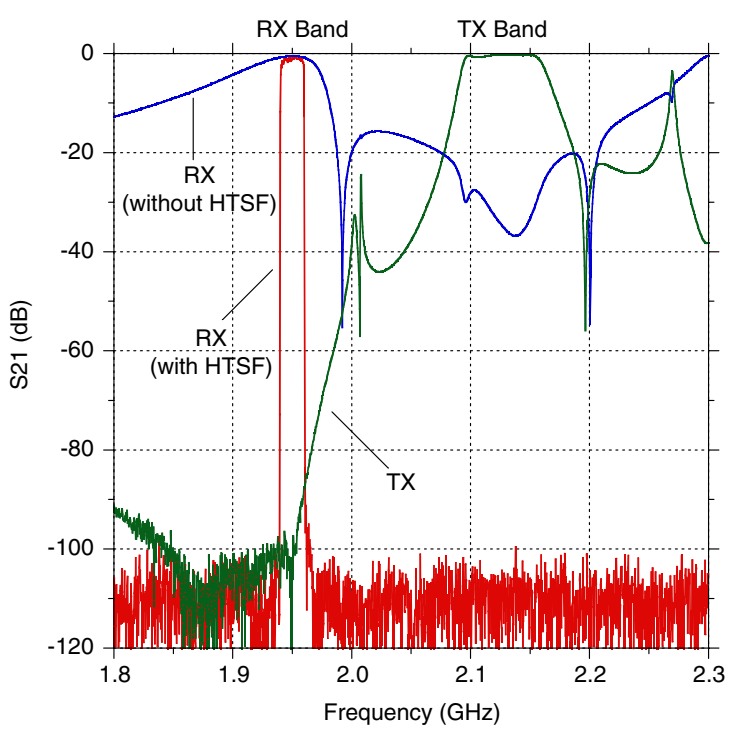

Figure 12. Frequency responses of the modified duplexer (duplexer only and with the HTSF).

suppress the intermodulation distortion generation. Figure 12 shows the frequency responses of the modified duplexer only and with the HTSF. The combination of the modified duplexer and HTSF achieves over $100 \mathrm{~dB}$ of entire TX band rejection.

\section{Total characteristics}

Figure 13 shows an experimental $2 \mathrm{GHz}$ band CRFE. It weighs $19 \mathrm{~kg}$ and occupies $35 \mathrm{l}$. The CRFE also utilizes heat pipes that efficiently remove the heat generated by the cryostat even when it is enclosed in a water-repellent enclosure. Efficient cooling can be achieved even under very severe environmental conditions at the top of most towers [12]. The CRFE has a volume of 151 (excluding heat pipes), which is $60 \%$ smaller than that previously proposed [3]. Table 1 summarizes principal characteristics for each component of 


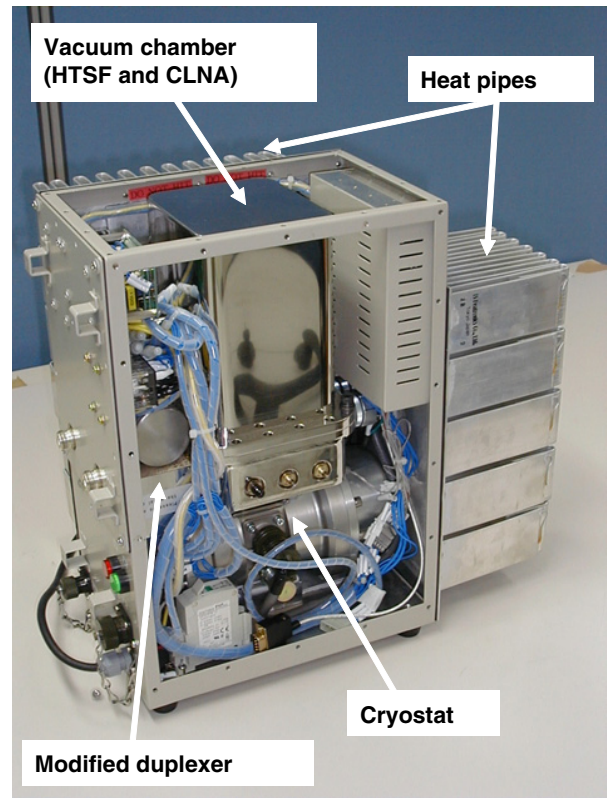

Figure 13. Photograph of the experimental CRFE.

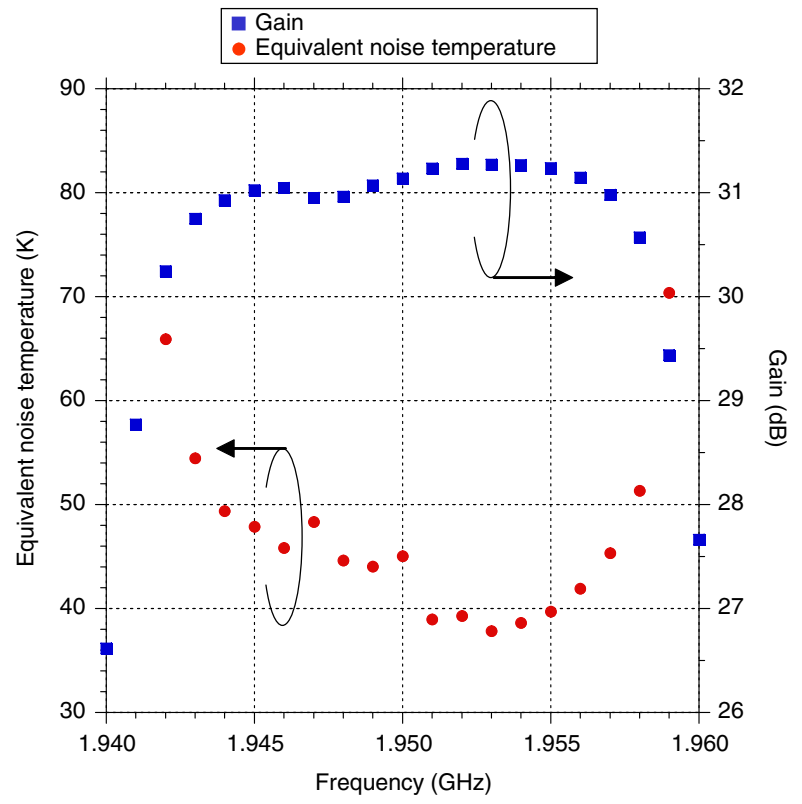

Figure 14. Equivalent noise temperature and gain of the experimental CRFE.

Table 1. Principal characteristics for each component of the CRFE.

\begin{tabular}{lll}
\hline HTSF & $\begin{array}{l}\text { Centre frequency: } 1.95 \mathrm{GHz} \\
\\
\end{array}$ & Passband width: $20 \mathrm{MHz}$ \\
& Insertion loss: $<0.5 \mathrm{~dB}$ & \\
CLNA & $\begin{array}{l}\text { Passband: } 1.9-2.0 \mathrm{GHz} \\
\text { Gain: } 30 \mathrm{~dB}\end{array}$ & \\
& $\begin{array}{l}\text { Equivalent noise temperature: }<25 \mathrm{~K} \\
\text { Cryostat }\end{array}$ & $\begin{array}{l}\text { Cooling capability: } 2 \mathrm{~W}(77 \mathrm{~K}) \\
\text { Durability: five-year maintenance free } \\
\text { (estimated) }\end{array}$ \\
\hline
\end{tabular}

the CRFE. In this section, all characteristics were measured at $70 \mathrm{~K}$. Figure 14 shows the measured equivalent noise

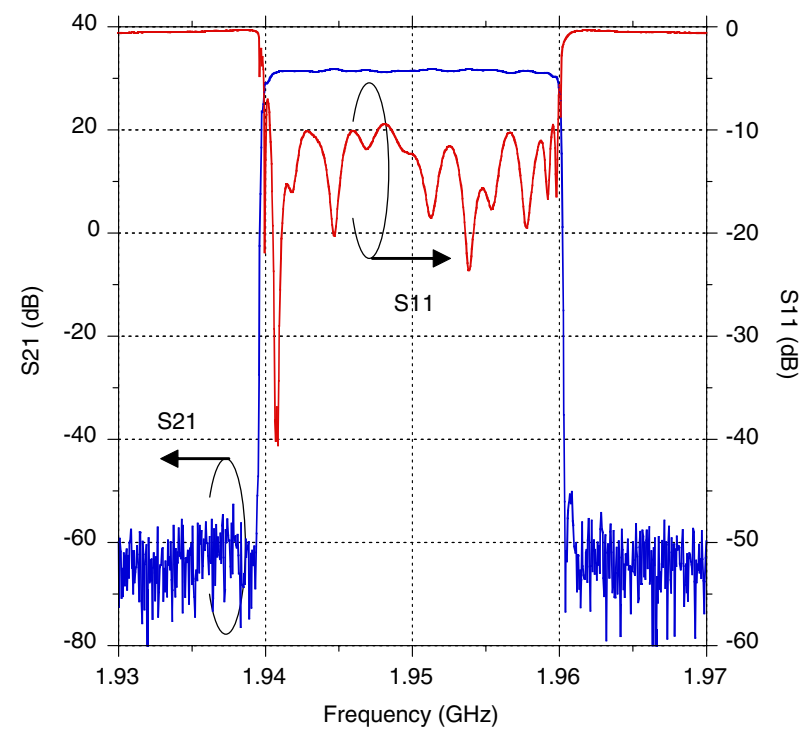

Figure 15. Frequency characteristics of the experimental CRFE.

temperature and gain of the CRFE. The CRFE has a $31.3 \mathrm{~dB}$ average passband gain and average noise temperature of $47.9 \mathrm{~K}$. Figure 15 shows the frequency characteristics of the experimental CRFE. The experimental CRFE has a centre frequency of $1.95 \mathrm{GHz}$, a passband width of $20 \mathrm{MHz}$, and a sharp skirt characteristic of $20 \mathrm{~dB} / 100 \mathrm{kHz}$. This device also has a $1.4 \mathrm{~dB}$ ripple for a $19 \mathrm{MHz}$ bandwidth (excluding band edges).

\section{Conclusion}

An experimental $2 \mathrm{GHz}$-band CRFE was constructed using an HTSF and CLNA to confirm sharp skirt characteristics. The improvement in receiver sensitivity due to CRFE use can be estimated to exceed $2.5 \mathrm{~dB}$ if the equivalent noise temperature lies in the range $255-272 \mathrm{~K}$. The experimental CRFE with sharp skirt characteristics is small and highly reliable, which ensures easy installation and maintenance because it is installed at the top of towers. For practical use, the CRFE must be resilient to all weather conditions such as lightning surges, corrosion, and wind pressure loads.

\section{References}

[1] Narahashi S, Mimura T and Nojima T 1997 IEICE Tech. Report vol SAT97-58, pp 57-63

[2] Braginski A I 1999 IEEE Trans. Appl. Supercond. 9 2825-36

[3] Nojima T, Narahashi S, Mimura T, Satoh K and Suzuki Y 2000 IEICE Trans. Commun. 83 1834-43

[4] Taub H and Schilling D L 1986 Principles of Communication Systems (New York: McGraw-Hill) pp 621-6

[5] Uebayashi S, Ohno K, Nojima T, Murata M and Yamada Y 1991 NTT R\&D 10 1309-18

[6] Tsuzuki G, Ye S and Berkowitz S 2003 IEEE Trans. Appl. Supercond. 2 261-4

[7] Abe Y, Kosuga H and Nitahara A 1990 3rd Asia-Pacific Microwave Conf. Proc. vol 28-6, pp 665-8

[8] Mimura T, Narahashi S and Nojima T 1997 IEICE Gen. Conf. vol B-5-143 
[9] Mimura T, Narahashi S and Nojima T 1997 IEICE Soc. Conf. vol B-5-153

[10] Okumura N 1997 MWE97 Microwave Workshop Dig. vol PS4-5, pp 332-5
[11] Hanes M, Chase D and Baid A O 1999 Cryocoolers vol 10 (New York: Plenum) pp 111-8

[12] Satoh K, Mimura T and Narahashi S 1998 IEICE Soc. Conf. vol B-5-44 